Pacific Journal of Mathematics

ASYMPTOTICITY AND SEMIMODULARITY IN PROJECTION 


\title{
ASYMPTOTICITY AND SEMIMODULARITY IN PROJECTION LATTICES
}

\author{
DAVID M. TopPING
}

\begin{abstract}
In this note it is shown that every von Neumann algebra has a semimodular projection lattice.

The notions of a dual modular pair of projections and nonasymptoticity, which were shown by Mackey to coincide in a type $I_{\infty}$ factor, are compared globally. A von Neumann algebra containing no asymptotic pairs of projections is characterized as the direct-sum of an abelian algebra and a finitedimensional algebra.
\end{abstract}

Two projections $e$ and $f$ in a von Neumann algebra $A$ are said to form a dual modular pair (relative to $A$ ), written $(e, f) D M$, if $(e \cup f) \cap g=e \cup(f \cap g)$ whenever $g$ is any projection in $A$ with $g \geqq e$. This notion, of course, makes sense in any lattice. A modular lattice is one in which every pair is a dual modular pair. According to current fashion, an orthocomplemented lattice (with 0 and 1 ) is called orthomodular if each element forms a dual modular pair with its orthocomplement. Following this trend, an orthomodular lattice is termed semimodular if the relation of dual modularity is symmetric [6] (p. 402).

The importance of semimodularity in the theory of orthomodular lattices became apparent when Mackey discovered that two projections $e$ and $f$ form a dual modular pair in the ring of all bounded operators if and only if the vector sum of $e$ and $f$ (we confuse projections with the subspaces onto which they project) is a closed subspace of the underlying Hilbert space. From this, it is immediate that every type I factor has a semimodular projection lattice.

Recent work of Ramsay [7] (p. 25, Theorem 5.4) shows that any dimension lattice having no type III summand is semimodular. This result specializes at once to show that the projection lattice of a semifinite von Neumann algebra is semimodular. In the other direction, MacLaren [6] has made an excellent case for semimodular lattices by deriving dimension lattice structure from semimodularity assumptions.

The case of type III has continued to be shrouded in mystery, largely because semimodularity is traditionally associated with the existence of dimension functions and covering conditions. We show in $\S 2$ that every von Neumann algebra has a semimodular projection lattice. A primary tool in our proof is the characterization of dual modular pairs given by Foulis [4] (p. 81, Theorem 28) in the context of Baer *-semigroups. 
A natural objection arises: What (if any) importance can be attached to semimodularity in the case of a type III dimension lattice, where modularity and dimension functions are so obviously lacking? The answer, we believe, lies in marking out the boundaries of a "more general lattice project" (i.e. more general than von Neumann's continuous geometries) suggested by Kaplansky. It seems desirable to know whether one should assume semimodularity in trying to bring some order to (for example) the chaotic perspectivity structure of the general orthomodular lattice, especially in view of recent developments (e.g. Fillmore's theorem on the transitivity of perspectivity in a von Neumann algebra [3], and MacLaren's atomic horizontal sums [6], where semimodularity fails).

Thus our contention is that semimodularity is a reasonable assumptions, at least if one agrees that projection lattices of von Neumann algebras are the correct models on which orthomodular geometry should be based.

Two projections $e$ and $f$ in a type $I_{\infty}$ factor form a dual modular pair if and only if $e$ and $f$ are not asymptotic (i.e. the "angle between $e$ and $f$ " is not zero). The situation in a general von Neumann algebra is not so simple. For instance, in a finite continuous algebra (type $\mathrm{II}_{1}$ ), asymptotic pairs abound and all such pairs are dual modular. We give a structure theorem for von Neumann algebras with no asymptotic pairs in $\S 4$.

The author wishes to thank Peter Fillmore and Arlan Ramsay for several stimulating discussions of semimodularity. He is especially grateful to D. J. Foulis for suggesting the description of dual modular pairs in Lemma 2 below.

2. Semimodularity. Throughout, $A$ will denote a von Neumann algebra. We call $A$ (or more precisely, its projection lattice) semimodular if $(e, f) D M$ implies $(f, e) D M$, for any two projections $e, f \in A$. For $x \in A$ we write $\mathscr{L}(x)$ and $\mathscr{R}(x)$ for the left and right annihilators, respectively, of $x$. We denote by $L P(x)$ the orthogonal projection on the closure of the range of $x$, and by $R P(x)$ the orthogonal projection on the orthogonal complement of the null space of $x$. Thus if $p=L P(x)$, then $p A=\mathscr{R}(\mathscr{L}(x))$; and if $p=R P(x)$, we have $A p=$ $\mathscr{L}(\mathscr{R}(x))$.

Lemma 1. For any two projections $e$ and $f$ in a von Neumann algebra $A$, there is a symmetry (=s.a. unitary) $s \in A$ such that $s(e f) s=f e$.

Proof. Let $a=e+f-1$ and set 


$$
s=R P\left(a^{+}\right)-R P\left(a^{-}\right)+(1-R P(a)),
$$

where $a^{ \pm}=\frac{1}{2}(|a| \pm a)$ and $|a|=\left(a^{2}\right)^{1 / 2}$. Then $s^{2}=1$ and $a=s|a|$. Now $e$ and $f$ commute with $|a|^{2}=a^{2}$ and since a positive operator and its square root commute with the same operators, $e$ and $f$ commute with $|a|$. Also $|a|=s a=s e-s(1-f)$ and hence $s e f=s a f=|a| f=$ $f|a|=f e s$ so that $s(e f) s=f e$.

In his extensive studies of Baer *-semigroups, D. J. Foulis has introduced the notion of a "range-closed" element [4]. In a von Neumann algebra $A$, we say that $x \in A$ is range-closed (relative to $A$ ) if for any projection $g \in A, A g \subset \mathscr{L}(\mathscr{R}(x))$ and $\mathscr{L}(x g)=\mathscr{L}(x)$ imply that $A g=\mathscr{L}(\mathscr{R}(x))$. The importance of this notion is clear from:

Lemma 2 (Foulis). For two projections e and $f$ in a von Neumann algebra $A$, the following are equivalent:

(1) $(e, f) D M$ relative to $A$.

(2) $f(1-e)$ is range-closed relative to $A$.

For the proof, we refer to Foulis' paper [4] (p. 81, Theorem 28).

We shall also need a result of E. A. Schreiner [8] (Theorem 9) which shows that an (apparently) stronger kind of symmetry implies semimodularity. It is this stronger property that we shall show holds for von Neumann algebras.

Lemma 3 (Schreiner). Let $L$ be any orthomodular lattice. If $(e, f) D M$ implies $(1-f, 1-e) D M$, for all $e, f \in L$, then $L$ is semimodular.

Putting these facts together, we have

THEOREM 1. The projection lattice of any von Neumann algebra is semimodular.

Proof. By Lemmas 2 and 3, it is enough to show that ef is range-closed implies that $f e$ is range-closed.

According to Lemma 1 , there is a symmetry $s \in A$ with $s(e f) s=f e$. The mapping $\theta$ from $A$ to itself defined by $\theta(x)=s x s$ for $x \in A$, is a *-automorphism of $A$ taking ef to $f e$. But any *-automorphism takes range-closed operators to range-closed operators (this simple observation was supplied by the referee) and the proof is complete.

3. An application. All remarks in this section are valid in any semimodular orthomodular lattice, but we prefer to phrase them in the language of operator algebras. The following simple fact is basic. 
Lemma 4. Two commuting projections in a von Neumann algebra form a dual modular pair.

Proof. Given projections $e, f \in A$ with $e f=f e$ and $g \in A$ a projection with $g \geqq e$. Then

$$
(e \cup f) \cap g=(e+f-e f) \cap g=((e-e f)+f) \cap g .
$$

But $(e-e f) \perp f$, so $(e-e f, f) D M$. Also $g \geqq e \geqq e-e f$ and hence

$$
((e-e f)+f) \cap g=e(1-f)+f \cap g \leqq e \cup(f \cap g)
$$

as required.

In particular, if $e \leqq f$, then $(e, f) D M$.

Lemma 5. If $(e, g) D M,(f, g) D M$ and either $(e, f \cup g) D M$ or $(f, e \cup g) D M$, then $(e \cup f, g) D M$.

Proof. Let $h \geqq e \cup f$. In case $(e, f \cup g) D M$ we have

$$
\begin{aligned}
& ((e \cup f) \cup g) \cap h=e \cup((f \cup g) \cap h) \\
& \quad=e \cup(f \cup(g \cap h))=(e \cup f) \cup(g \cap h),
\end{aligned}
$$

so $(e \cup f, g) D M$. In case $(f, e \cup g) D M$ we have

$$
\begin{aligned}
& ((e \cup f) \cup g) \cap h=f \cup((e \cup g) \cap h) \\
& \quad=f \cup(e \cup(g \cap h))=(e \cup f) \cup(g \cap h)
\end{aligned}
$$

and again we conclude $(e \cup f, g) D M$.

The next lemma shows that dual modular pairs can be "added" in case enough orthogonality is present.

LEMMA 6. If $\left(e_{i}, f_{i}\right) D M(i=1,2)$ and if $\left(e_{1} \cup f_{1}\right) \perp\left(e_{2} \cup f_{2}\right)$, then $\left(e_{1} \cup e_{2}, f_{1} \cup f_{2}\right) D M$.

Proof. Let $e=e_{1} \cup e_{2}$ and $f=f_{1} \cup f_{2}$. Since $e_{2} \perp\left(e_{1} \cup f_{1}\right)$ we have $\left(e_{2}, e_{1} \cup f_{1}\right) D M$ and the relations $\left(e_{1}, f_{1}\right) D M$ and $\left(e_{2}, f_{1}\right) D M$ together with Lemma 5 show that $\left(e, f_{1}\right) D M$. Similarly, $\left(e, f_{2}\right) D M$. By semimodularity (Theorem 1), $\left(f_{i}, e\right) D M(i=1,2)$. Next we assert that $\left(e \cup f_{2}, f_{1}\right) D M$. For if $h \geqq e \cup f_{2}$ then $h \geqq e_{2} \cup f_{2}$ and

$$
\begin{gathered}
\left(\left(e \cup f_{2}\right) \cup f_{1}\right) \cap h=\left(\left(e_{2} \cup f_{2}\right) \cup\left(e_{1} \cup f_{1}\right)\right) \cap h \\
=\left(e_{2} \cup f_{2}\right) \cup\left(\left(e_{1} \cup f_{1}\right) \cap h\right)
\end{gathered}
$$

by the orthogonality hypothesis. Since $h \geqq e_{1}$, this last expression reduces to 


$$
\left(e_{2} \cup f_{2}\right) \cup\left(e_{1} \cup\left(f_{1} \cap h\right)\right)=\left(e \cup f_{2}\right) \cup\left(f_{1} \cap h\right)
$$

as desired. Free use of semimodularity and a final application of Lemma 5 gives $(e, f) D M$.

Two projections $e$ and $f$ are said to be in position $p^{\prime}$ if $e \cap(1-f)=$ $0=(1-e) \cap f$. The proof of the next lemma, an exercise in the use of orthomodularity, is left to the reader.

LEMma 7. Any two projections $e$ and $f$ can be decomposed orthogonally into $e=e_{1}+e_{2}$ and $f=f_{1}+f_{2}$ where:

(1) $e_{1}$ and $f_{1}$ are in position $p^{\prime}$;

(2) $e_{2} \perp f_{2}$; and

(3) $\left(e_{1} \cup f_{1}\right) \perp\left(e_{2} \cup f_{2}\right)$.

Explicitly, $e_{1}=e-e \cap(1-f), f_{1}=f-(1-e) \cap f, e_{2}=e \cap(1-f)$ and $f_{2}=(1-e) \cap f$.

Our application is a new characterization of finite von Neumann algebras.

Proposition 1. For a von Neumann algebra $A$, the following are equivalent:

(1) $A$ is of finite class.

(2) Any two projections $e, f \in A$ in position $p^{\prime}$ form a dual modular pair.

Proof. Since $A$ is finite if and only if its projection lattice is modular, (2) clearly follows from (1). For the converse implication, one applies the decomposition of Lemma 7 and then the addition property of Lemma 6 .

4. Asymptotic projections. Two projections $e$ and $f$ with $e \cap f=0$ are asymptotic, written $e \| f$, if $\sup |(\alpha \mid \beta)|=1$, where $\|\alpha\|=1=\|\beta\|, \alpha \in e, \beta \in f$. We list some classical results surrounding this notion. A good historical account of these ideas can be found in [1] (p. 21, §5).

Lemma 8 (Murray, Lorch, Kober, Mackey). For two projections $e$ and $f$ with $e \cap f=0, e \# f$ if and only if the vector sum

$$
\{\alpha+\beta: \alpha \in e, \beta \in f\}
$$

is closed.

LEMMA 9 (Mackey [5]). The vector sum of $e$ and $f$ is closed if and only if $(e, f) D M$ relative to the ring of all bounded operators. 
Lemma 9 of course implies semimodularity in the case of a type $I_{\infty}$ factor.

Corollary 1. If $e \# f$, then $(e, f) D M$ relative to any von Neumann algebra $A$ containing $e$ and $f$.

If $e$ and $f$ are nonzero projections with $e \cap f=0$, let $\cos (e, f)=$ $\sup |(\alpha \mid \beta)|$, where $\|\alpha\|=1=\|\beta\|, \alpha \in e, \beta \in f$. In case $e \# f$ (i.e. $\cos (e, f)<1$ ) we have $\cos ^{2}(e, f)=1-\left\|p_{e, f}\right\|^{2}$, where $p_{e, f}$ is the projection (not necessarily self-adjoint, but bounded) onto $e$ along $f$. The idempotents $p_{e, f}$ and $p_{f, e}$ are characterized by the algebraic properties: (i) $e p_{e, f}(e \cup f)=p_{e, f}$, (ii) $f p_{f, e}(e \cup f)=p_{f, e}$, and (iii) $p_{e, f}+p_{f, e}=e \cup f$. Conversely, if two bounded idempotent operators exist with these properties, then $e \cup f$ is the vector sum of $e$ and $f$, so that $e \# f$. These results, as well as the next lemma, can be found in Feldman's thesis [2] (p. 13, Theorem 3 and its Corollary).

LEMma 10 (Feldman). Asymptoticity and the function cos (, ) are invariants of *-isomorphisms between von Neumann algebras.

The suggestive terminology in the next proposition will be clarified in the proof.

Proposition 2. A von Neumann algebra is nonabelian if and only if it contains arbitrary small angles.

Proof. Suppose first that $A$ is nonabelian and let $e, f \in A$ be two orthogonal equivalent projections. Let $x \in A$ be a partial isometry implementing the equivalence: $e=x^{*} x$ and $f=x x^{*}$. Take $\alpha \in e$ and $\beta \in f$ with $\beta=x \alpha,\|\alpha\|=1=\|\beta\|$ and let $0<\lambda, \mu<1$ with $\lambda^{2}+\mu^{2}=$ 1. Define $\gamma=\left(\frac{1}{2}(1+\lambda)\right)^{1 / 2} \alpha+\mu(2(1+\lambda))^{-1 / 2} \beta$ and

$$
g=\frac{1}{2}\left[(1+\lambda) e+\mu\left(x+x^{*}\right)+(1-\lambda) f\right] \text {. }
$$

A straightforward calculation shows that $g$ is a projection, $\gamma \in g$, $\|\gamma\|=1$ and $(\alpha \mid \gamma)=\left(\frac{1}{2}(1+\lambda)\right)^{1 / 2}$. Hence by choosing $\lambda$ close to 1 , we can make $\cos (e, g)$ close to 1 .

Finally, if $A$ is abelian, then $e \cap f=0$ forces $e \perp f$, so in fact the only nonzero "angles" are "right angles."

The next lemma provides a simple means of constructing asymptotic pairs.

LEMMA 11. If a von Neumann algebra contains an infinite orthogonal sequence of nonabelian projections, then it also contains a pair of asymptotic projections. 
Proof. Let $\left\{p_{i}\right\}_{i=1}^{\infty}$ be an infinite orthogonal sequence of nonabelian projections in $A$. Then each algebra $p_{i} A p_{i}$ contains a $2 \times 2$ matrix algebra and hence by Proposition 2 we can find two projections $e_{i}, f_{i} \leqq p_{i}$ with $e_{i} \cap f_{i}=0$ and a pair of unit vectors $\alpha_{i} \in e_{i}, \beta_{i} \in f_{i}$ for which $\left(\alpha_{i} \mid \beta_{i}\right) \geqq 1-(i+1)^{-1}$. Putting $e=\operatorname{LUB} e_{i}$ and $f=\operatorname{LUB} f_{i}$ we obtain an asymptotic pair of projections in $A$.

The final link in our argument is provided by

Proposition 3. Let $Z$ be an abelian von Neumann algebra and let $A$ be the algebra of all $n \times n$ matrices ( $n$ finite $\geqq 2$ ) with entries from $Z$. Then $A$ has no asymptotic pairs if and only if $Z$ is finitedimensional.

Proof. If $Z$ is finite-dimensional, so is $A$ and hence $A$ is *isomorphic to an algebra on a finite-dimensional space. Since an algebra of the latter type clearly has no asymptotic pairs, we conclude from Lemma 10 that $A$ has none either.

Conversely, if $Z$ is infinite-dimensional, it contains an infinite orthogonal sequence $\left\{\varepsilon_{i}\right\}_{i=1}^{\infty}$ of nonzero projections. If $e_{i}$ is the matrix in $A$ having $\varepsilon_{i}$ in each diagonal position and zeros elsewhere, then $\left\{e_{i}\right\}_{i=1}^{\infty}$ is an orthogonal sequence of (nonabelian) central projections in $A$, so by Lemma 11, $A$ contains an asymptotic pair of projections.

Assembling the above results, we obtain the structure theorem promised earlier.

THEOREm 2. For a von Neumann algebra A, the following are equivalent:

(1) A contains no asymptotic pair of projections.

(2) $A$ is the direct-sum of an abelian algebra and a finitedimensional algebra.

Proof. First note that if $A$ has no asymptotic pairs, then it cannot contain an infinite orthogonal sequence of nonabelian projections by Lemma 11. If $A$ had a non type I portion, the "Halving Lemma" (see e.g. [9], p. 34, Theorem 17) would yield a sequence of the type mentioned, so $A$ must be type I, and, in fact, of finite class by Corollary 1 and Proposition 1.

Next we observe that $A$ has a largest central abelian projection, so we may split off the abelian summand determined thereby. Assuming now that $A$ has no abelian summand, the known structure theory for type I algebras may be applied to decompose $A$ into a direct-sum of homogeneous algebras. Since each homogeneous summand is nonabelian, the number of summands is finite by Lemma 11. Finally, each homo- 
geneous summand is an $n \times n$ matrix algebra ( $n$ finite $\geqq 2$ ) over its center, and the latter must be finite-dimensional by Proposition 3 . Therefore (1) implies (2).

The converse implication is contained in Proposition 3 and the last paragraph of the proof of Proposition 2.

Corollary 2. Any von Neumann algebra whose nonabelian summand contains an infinite-dimensional summand of finite class has a dual modular pair of asymptotic projections.

The method used in the proof of Theorem 2 yields the converse of Lemma 11, which can be regarded as the global version of a result of Dixmier [1] (Proposition 1.1 b., p. 21).

Corollary 3. If a von Neumann algebra contains a pair of asymptotic projections, then it also contains an infinite orthogonal sequence of nonabelian projections.

Ramsay has asked whether a type III algebra can have noncommuting dual modular pairs. It is easy to find such pairs in any nonabelian algebra. For as in Proposition 2, we can drop down to a $2 \times 2$ matrix subalgebra and find projections $e$ and $f$ with $e \cap f=0$ and, e.g., $\cos (e, f)=\frac{1}{2}$. Then $e \# f$, and by Corollary $1,(e, f) D M$. However $e$ and $f$ cannot commute, for this would imply $e \perp f$, since $e \cap f=0$.

The semimodularity result can also be extended to $A W^{*}$-algebras, $B_{p}^{*}$-algebras $\left(C^{*}\right.$-algebras containing the left and right projections of each operator) and $J W$-algebras [9]. In a $J W$-algebra $A$, one calls fef range-closed if for any projection $g \in A,\{f e f\}^{\perp} \subset\{g\}^{\perp}$ and $\{e f g f e\}^{\perp}=$ $\{e f e\}^{\perp}$ imply that $\{f e f\}^{\perp}=\{g\}^{\perp}$. One then proves the Jordan analog of Lemma 2. Using Proposition 6 of [9] one obtains a symmetry $s \in A$ with $s(e f e) s=f e f$ and proceeds much as before. We omit the details.

The author is indebted to the referee for pointing out an oversight in Theorem 2 and for an example suggesting Proposition 3.

\section{REFERENCES}

1. J. Dixmier, Étude sur les variétés et les opérateurs de Julia, avec quelques applications, Bull. Soc. Math. France 77 (1949), 11-101.

2. J. Feldman, Isomorphisms of rings of operators, Thesis, University of Chicago, 1954 .

3. P. Fillmore, Perspectivity in projection lattices, Proc. Amer. Math. Soc. 16 (1965), 383-387. 
4. D. Foulis, Relative inverses in Baer *-semigroups, Mich. Math. J. 10 (1963), 65-84.

5. G. Mackey, On infinite dimensional linear spaces, Trans. Amer. Math. Soc. 57 (1945), 155-207.

6. M. MacLaren, Nearly modular orthocomplemented lattices, Trans. Amer. Math. Soc. 114 (1965), 401-416.

7. A. Ramsay, Dimension theory in complete orthocomplemented weakly modular lattices, Trans. Amer. Math. Soc. 116 (1965), 9-31.

8. E. Schreiner, Modular pairs in orthomodular lattices (to appear in Pacific J. Math.)

9. D. Topping, Jordan algebras of self-adjoint operators, Amer. Math. Soc. Memoirs No. 53 (1965).

Received February 21, 1966.

UNIVERSITY OF WASHINGTON 



\section{PACIFIC JOURNAL OF MATHEMATICS}

\section{EDITORS}

\author{
H. SAMELSON \\ Stanford University \\ Stanford, California \\ J. P. JANS \\ University of Washington \\ Seattle, Washington 98105
}

\section{J. DugundJI}

University of Southern California Los Angeles, California 90007

RICHARD ARENS

University of California

Los Angeles, California 90024

\section{ASSOCIATE EDITORS}
E. F. BECKENBACH
B. H. NeumanN
F. WOLF
K. YOSIDA

\section{SUPPORTING INSTITUTIONS}

\author{
UNIVERSITY OF BRITISH COLUMBIA \\ CALIFORNIA INSTITUTE OF TECHNOLOGY \\ UNIVERSITY OF CALIFORNIA \\ MONTANA STATE UNIVERSITY \\ UNIVERSITY OF NEVADA \\ NEW MEXICO STATE UNIVERSITY \\ OREGON STATE UNIVERSITY \\ UNIVERSITY OF OREGON \\ OSAKA UNIVERSITY \\ UNIVERSITY OF SOUTHERN CALIFORNIA
}

\author{
STANFORD UNIVERSITY \\ UNIVERSITY OF TOKYO \\ UNIVERSITY OF UTAH \\ WASHINGTON STATE UNIVERSITY \\ UNIVERSITY OF WASHINGTON \\ AMERICAN MATHEMATICAL SOCIETY \\ CHEVRON RESEARCH CORPORATION \\ TRW SYSTEMS \\ NAVAL ORDNANCE TEST STATION
}

Mathematical papers intended for publication in the Pacific Journal of Mathematics should be typewritten (double spaced). The first paragraph or two must be capable of being used separately as a synopsis of the entire paper. It should not contain references to the bibliography. Manuscripts may be sent to any one of the four editors. All other communications to the editors should be addressed to the managing editor, Richard Arens at the University of California, Los Angeles, California 90024 .

50 reprints per author of each article are furnished free of charge; additional copies may be obtained at cost in multiples of 50 .

The Pacific Journal of Mathematics is published monthly. Effective with Volume 16 the price per volume (3 numbers) is $\$ 8.00$; single issues, $\$ 3.00$. Special price for current issues to individual faculty members of supporting institutions and to individual members of the American Mathematical Society: $\$ 4.00$ per volume; single issues $\$ 1.50$. Back numbers are available.

Subscriptions, orders for back numbers, and changes of address should be sent to Pacific Journal of Mathematics, 103 Highland Boulevard, Berkeley 8, California.

Printed at Kokusai Bunken Insatsusha (International Academic Printing Co., Ltd.), No. 6, 2-chome, Fujimi-cho, Chiyoda-ku, Tokyo, Japan.

PUBLISHED BY PACIFIC JOURNAL OF MATHEMATICS, A NON-PROFIT CORPORATION

The Supporting Institutions listed above contribute to the cost of publication of this Journal, but they are not owners or publishers and have no responsibility for its content or policies. 


\section{Pacific Journal of Mathematics \\ Vol. 20, No. $2 \quad$ October, 1967}

Edward Dewey Davis, Ideals of the principal class, $R$-sequences and a certain monoidal transformation ............................. 197

Richard Mansfield Dudley, Sub-stationary processes ................ 207

Newton Seymour Hawley and M. Schiffer, Riemann surfaces which are doubles of plane domains ......................... 217

Barry E. Johnson, Continuity of transformations which leave invariant certain translation invariant subspaces ................... 223

John Eldon Mack and Donald Glen Johnson, The Dedekind completion of

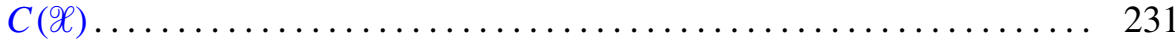

K. K. Mathur and R. B. Saxena, On the convergence of quasi-Hermite-Fejér interpolation................................... 245

H. D. Miller, Generalization of a theorem of Marcinkiewicz............ 261

Joseph Baruch Muskat, Reciprocity and Jacobi sums ............... 275

Stelios A. Negrepontis, On a theorem by Hoffman and Ramsay .......... 281

Paul Adrian Nickel, A note on principal functions and multiply-valent canonical mappings .............................. 283

Robert Charles Thompson, On a class of matrix equations ............. 289

David Morris Topping, Asymptoticity and semimodularity in projection lattices ........................................ 317

James Ramsey Webb, A Hellinger integral representation for bounded linear functionals

Joel John Westman, Locally trivial $C^{r}$ groupoids and their representations...

Hung-Hsi Wu, Holonomy groups of indefinite metrics 